\title{
New formulations for the hop-constrained minimum spanning tree problem via Sherali and Driscoll's tightened Miller-Tucker-Zemlin constraints
}

\author{
İbrahim Akgün* \\ Department of Industrial Engineering, Bilkent University, Bilkent 06800, Ankara, Turkey
}

\section{A R T I C L E I N F O}

Available online 11 May 2010

\section{Keywords:}

Miller-Tucker-Zemlin constraints

Spanning trees

Network flows

Integer programming

Hop constraints

\begin{abstract}
A B S T R A C T
Given an undirected network with positive edge costs and a natural number $p$, the hop-constrained minimum spanning tree problem (HMST) is the problem of finding a spanning tree with minimum total cost such that each path starting from a specified root node has no more than $p$ hops (edges). In this paper, the new models based on the Miller-Tucker-Zemlin (MTZ) subtour elimination constraints are developed and computational results together with comparisons against MTZ-based, flow-based, and hop-indexed formulations are reported. The first model is obtained by adapting the MTZ-based Asymmetric Traveling Salesman Problem formulation of Sherali and Driscoll [18] and the other two models are obtained by combining topology-enforcing and MTZ-related constraints offered by Akgün and Tansel (submitted for publication) [20] for HMST with the first model appropriately. Computational studies show that the best LP bounds of the MTZ-based models in the literature are improved by the proposed models. The best solution times of the MTZ-based models are not improved for optimally solved instances. However, the results for the harder, large-size instances imply that the proposed models are likely to produce better solution times. The proposed models do not dominate the flowbased and hop-indexed formulations with respect to LP bounds. However, good feasible solutions can be obtained in a reasonable amount of time for problems for which even the LP relaxations of the flowbased and hop-indexed formulations can be solved in about 2 days.
\end{abstract}

(c) 2010 Elsevier Ltd. All rights reserved.

\section{Introduction}

Minimum spanning tree problems arise quite naturally in transportation and communication network design when it is necessary to provide a minimum-cost connectivity among a number of geographically dispersed locations or system components. Various examples of minimum cost tree networks are given by Ahuja et al. [1]. We consider in this paper a topology constrained version of the minimum spanning tree problem in which a minimum cost spanning tree is sought for while requiring that each path from a specified root node to every other node is required to have at most a fixed number of hops (edges).

Hop requirements may arise in the design of centralized communication networks where a number of sites (computers/ terminals or switching sites) need to be connected to a central unit that is linked to a backbone network. In such a network, the central unit acts as the root node. A hop constraint is imposed to ensure a certain level of service quality between the root node and all other nodes. For example, a delay constraint may be thought of as a hop constraint because the total delay is dependent on the

\footnotetext{
* Fax: +90312 2664054 .

E-mail address: iakgun@bilkent.edu.tr
}

number of intermediate nodes on the path from the root to another node. A reliability constraint, i.e., the probability that the service will not be terminated by the failure of an intermediate node/link, may also be represented as a hop constraint because the reliability of a network increases as the number of hops decreases in general. Woolston and Albin [2] show that the spanning-tree designs with hop limits perform better than the ones without hop limits with respect to the reliability measure. LeBlanc et al. [3], Balakrishnan and Altinkemer [4], and Gouveia et al. [5] discuss applications of hop constraints in more general network design problems. Dahl [6] also defines applications in transportation, statistics, and plant location for the case with $p=2$.

To define the problem of interest, let $G=(V, E)$ be an undirected connected network with node set $V=\{r, 1, \ldots, n\}$, edge set $E$, and positive edge costs $c_{e}(e \in E)$. A spanning tree of $G$ is a connected sub-graph of $G$ that has no cycles and spans all nodes. Given a natural number $p$, a spanning tree is a hop-constrained spanning tree if the unique path from a specified root node $r$ to any other node $i$ has no more than $p$ hops (edges). From now on, we refer to hop-constrained spanning trees as feasible trees. If $p \geq n$, all spanning trees are feasible trees and hop constraints can be ignored. If $p=1$, finding a feasible tree is trivial. The distinction between feasible and infeasible trees becomes important for 
$2 \leq p \leq n-1$ and hence this is assumed in the rest of the paper. We refer to the problem of finding a minimum cost spanning tree of $G$ as the minimum spanning tree (MST) problem and that of finding a minimum cost feasible tree as the hop-constrained minimum spanning tree (HMST) problem.

While MST is solvable in low order polynomial time by the algorithms of Kruskal [7] and Prim [8], HMST is NP-Hard because HMST is equivalent to the Uncapacitated Facility Location Problem for $p=2$, e.g., Gouveia [9] and Dahl [6]. Manyem and Stallmann [10] show HMST is not in APX, i.e., the class of problems for which it is possible to have polynomial time heuristics with a guaranteed approximation bound. Dahl [6] studies HMST with $p=2$ and compares the polyhedra of directed and undirected models. Alfandari and Paschos [11] show that this version of the problem cannot be approximated by polynomial time approximation schemes unless $\mathrm{P}=\mathrm{NP}$.

There are a number of exact and heuristic methods developed for HMST in the literature. In this study, the focus is on the formulation of HMST based on Miller-Tucker-Zemlin (MTZ) subtour elimination constraints [12].

Among different subtour elimination constraints, e.g., packing, cut-sets, and flows [13], constraints developed by Dantzig et al. [14] are known to give the best representation of ATSP polytope but at the expense of an exponential number of constraints. MTZ constraints are attractive due to their compactness. However, they are well-known for producing weak linear programming (LP) relaxation bounds. Orman and Williams [15] compare the strengths of eight different formulations of the asymmetric traveling salesman problem (ATSP) including the ones with MTZ constraints and flow constraints by their LP relaxation bounds. They find out that the LP relaxation polytope obtained by MTZ constraints contains some of the seven existing formulations. This fact has led to various studies that augment the MTZ constraints to strengthen the LP bounds, e.g., Desrochers and Laporte [16], Gouveia and Pires [17], and Sherali and Driscoll [18]. Desrochers and Laporte [16] lift the MTZ constraints into facets of the underlying ATSP polytope. Gouveia and Pires [17] develop a compact formulation based on a disaggregation of MTZ constraints whose LP relaxation is characterized by a set of circuit inequalities given by Grötschel and Padberg [19]. These circuit inequalities are then lifted into several different facet-defining inequalities. Sherali and Driscoll [18] apply the reformulationlinearization technique (RLT) to a nonstandard restatement of ATSP-MTZ including nonlinear product terms and obtain a new formulation. The resulting formulation ATSP-SD is compact and dominates the formulation of Desrochers and Laporte [16] both theoretically and computationally.

Although most studies about MTZ constraints focus on the TSP or TSP-related problems, the formulations or liftings in those studies can be adapted to other problems where subtours are not allowed. HMST is one of those problems. Gouveia [9] discusses HMST and presents several formulations based on MTZ constraints. Liftings to MTZ constraints, some of which are based on the liftings of Desrochers and Laporte [16], and lower bounding schemes based on the Lagrangean relaxation combined with subgradient optimization are offered. Computational results indicate that the lower bounds obtained by the LP relaxations of the models and the Lagrangean relaxation schemes are weak implying that new formulations with tighter LP bounds are needed.

Akgün and Tansel [20] propose new MTZ-based formulations for HMST. The formulations are based on a new set of topologyenforcing constraints, a new set of MTZ-related constraints, and the liftings to MTZ constraints offered by Gouveia [9]. Computational results indicate that the proposed formulations give better LP relaxation bounds and solution times than their corresponding ones in Gouveia [9] and that the new set of topology-enforcing and MTZ-related constraints is competitive with liftings to MTZ constraints in Gouveia [9], some of which are based on liftings of Desrochers and Laporte [16].

In this research, new MTZ-based models for HMST are presented. The new models are different from the previous ones because they use the ATSP model of Sherali and Driscoll [18] as the basis to formulate HMST. The first model is obtained by adapting ATSP model of Sherali and Driscoll [18]. The other two models are obtained by incorporating the topology-enforcing and MTZ-related constraints offered by Akgün and Tansel [20] for HMST into the first model appropriately. Computational studies show that the LP bounds of the previous MTZ-based models are improved while the best solution times are not for optimally solved problems. However, the results imply that the proposed models are likely to produce better solution times for the harder, large-size instances. The proposed models do not dominate the flow-based and hop-indexed formulations with respect to LP bounds. On the other hand, good feasible solutions can be obtained in seconds for problems for which even the LP relaxations of the flow-based and hop-indexed formulations can be solved in about 2 days.

Other than the MTZ-based studies, there is a body of literature on flow-based and hop-indexed formulations for HMST, e.g., Gouveia [21,22], Gouveia and Requejo [23], Balakrishnan and Altinkemer [4], Pirkul and Soni [24], and Gouveia et al. [5]. Even though the LP bounds are improved significantly over MTZ-based models in these studies, the formulations lead to very large LP models that may require huge computer storage requirements and excessive CPU times for high values of $p$ and for intermediate or larger size networks. Dahl et al. [25] offer a formulation using only natural design variables and an exponential number of constraints composed of the so-called jump inequalities shown to be facet-defining. The proposed formulation uses fewer variables but has weaker LP bounds than the ones based on flow-based models. Lagrangean-based bounding schemes are also offered in the aforementioned studies. Dahl et al. [26] summarize the previous studies. Kerivin and Mahjoub [27] give a survey of several network design problems with hop constraints and methods to solve them. Gouveia et al. [28] propose a modeling approach where the whole problem is viewed as defined in a single layered graph and modeled by using an exponential number of constraints. They show that the HMST is equivalent to a Steiner tree problem $[29,30]$ in an adequate layered graph. The proposed approach gives significantly better results than the previously known methods.

Other problems related to HMST are the Rooted DistanceConstrained MST Problem [31] and the Diameter-Constrained MST Problem, e.g., Gouveia et al. [28], Achuthan et al. [32], Gouveia and Magnanti [33], Gruber and Raidl [34], and Santos et al. [35].

The remainder of the paper is organized as follows. Section 2 gives ATSP formulation of Sherali and Driscoll [18], Section 3 develops a formulation for HMST by adapting the formulation in Section 2, Section 4 offers two new improved models, Section 5 gives computational studies, and Section 6 concludes the paper.

\section{ATSP formulation of Sherali and Driscoll [18]}

The $\operatorname{ATSP}$ (e.g., [36-38]) is defined on a directed graph $G=(V, A)$ where $V=\{1, \ldots, n\}$ is the vertex set and $A=\{(i, j): i, j \in V, i \neq j\}$ is the arc set. A cost of $c_{i j}$ is associated with each $\operatorname{arc}(i, j)$ with $c_{i j} \neq c_{j i}$ in general. The ATSP is then the problem of identifying a tour starting and ending at a base (root) node $r \in V$ and visiting each node once such that the total cost is minimized. 
In this section, two formulations are given for ATSP, one is ATSP-MTZ based on basic MTZ constraints [12] and the other is ATSP-SD based on SD constraints [18].

Model ATSP-MTZ: ATSP formulation based on basic MTZ constraints

$$
\begin{aligned}
& z^{*}=\min _{x, u} \sum_{(i, j) \in A} c_{i j} x_{i j} \\
& \text { s.t. } \quad \sum_{j \neq i} x_{i j}=1, \quad i \in V \\
& \sum_{i \neq j} x_{i j}=1, \quad j \in V \\
& u_{i}-u_{j}+(n-1) x_{i j} \leq n-2 \quad(i, j) \in A, i, j \neq r \\
& u_{i} \equiv 0, \quad i=r \\
& u_{i} \geq 1, \quad i \neq r \\
& u_{i} \leq n-1, \quad i \neq r \\
& u_{i} \geq 0 \forall i \\
& x_{i j} \in\{0,1\} \quad(i, j) \in A
\end{aligned}
$$

Two sets of decision variables are used in ATSP-MTZ: (1) binary variables $x_{i j}$ that take on the value of 1 if arc $(i, j)$ is in the design, i.e., arc $(i, j)$ is a part of the optimal tour, and 0 otherwise and (2) non-negative node-labeling variables $u_{i}$.

Objective function (1) minimizes the total cost of the tour. Constraints (2) and (3) are the standard assignment constraints. Constraints (4) through (7) are MTZ constraints. They eliminate all subtours that do not contain the root node $r$ by assigning unique labels $u_{i}$ to nodes such that the label of a node represents the rank-order in which the node is visited in a traveling salesman tour. That is, base node $r$ is assigned a label of 0 while the $i$ th node visited after node $r$ is assigned a label of $i$. Constraints (4) require that $u_{j} \geq u_{i}+1$ whenever $x_{i j}=1$ given that $j \neq r$. However, because $u_{i} \leq n-1$ by constraints (7) and there are $n$ nodes, $u_{j}=u_{i}+1$. Constraint (5) assigns a label value of zero to node $r$. Constraints (6) and (7) define lower and upper bounds on the label values of non-root nodes, respectively. Constraints (8) and (9) give the appropriate set restrictions and non-negativity on the decision variables.

Sherali and Driscoll [18] reformulate ATSP-MTZ by using nonlinear product terms and then apply a specialized version of RLT to that formulation to obtain ATSP-SD. The applied procedure is given next following the lines of Sherali and Driscoll [18].

\section{Model ATSP-MTZ1: Nonlinear formulation of ATSP-MTZ}

In addition to (1)-(2) and (5)-(9),

$u_{j} x_{i j}=\left(u_{i}+1\right) x_{i j}, \quad i, j \neq r, i \neq j$

$u_{j} x_{r j}=x_{r j}, \quad j \neq r$

$u_{j} x_{j r}=(n-1) x_{j r}, \quad j \neq r$

Constraints (5)-(7) and (10)-(12) are subtour elimination constraints. For $j \neq r, u_{j}=1$ if $x_{r j}=1$, and $u_{j}=n-1$ if $x_{j r}=1$. For $i$, $j \neq r, i \neq j, u_{j}=u_{i}+1$ if $x_{i j}=1$. These conditions together with (1), (2) and (9) eliminate any subtours that do not contain the root node (base city).

Reformulation phase: Additional sets of constraints are constructed via steps R1 through R4.

R1: Using constraints (2) and (3), construct valid inequalities $u_{i}\left[\sum_{j \neq i} x_{i j}-1\right]=0$ for $i \neq r$ and $u_{j}\left[\sum_{i \neq j} x_{i j}-1\right]=0$ for $j \neq r$.
R2: For each $j \neq r$, multiply $\left(u_{j}-1\right) \geq 0$ by (i) $x_{j i} \geq 0$ for $i \neq r, i \neq j$ and by (ii) $\left(1-x_{i j}-x_{j i}\right) \geq 0$ for $i \neq r, i \neq j$. These multiplications yield valid inequalities of type (i) $\left(u_{j}-1\right) x_{j i} \geq 0$ for $i, j \neq r, i \neq j$ and (ii) $\left(u_{j}-1\right)\left(1-x_{i j}-x_{j i}\right) \geq 0$ for $i, j \neq r, i \neq j$. Because $\left\{x_{i j}+x_{j i} \leq 1\right.$, $\left.x_{i j} \geq 0, x_{j i} \geq 0\right\}$ implies the set of constraints $\left\{0 \leq x_{i j} \leq 1,0 \leq x_{j i} \leq 1\right\}$, the use of $\left(1-x_{i j}-x_{j i}\right)$ generates potentially tighter relaxations than that obtained using $\left(1-x_{i j}\right)$ and $\left(1-x_{j i}\right)$.

R3: Similar to (R2), using (7), construct the valid inequalities (i) $\left(n-1-u_{j}\right) x_{i j} \geq 0$ for $i, j \neq r, \quad i \neq j$ and (ii) $\left(n-1-u_{j}\right)\left(1-x_{i j}-x_{j i}\right)$ $\geq 0$ for $i, j \neq r, i \neq j$.

R4: For $j \neq r$, construct the base city product constraints $\left(u_{j}-2\right)\left(1-x_{r j}-x_{j r}\right) \geq 0$ and $\left(n-2-u_{j}\right)\left(1-x_{r j}-x_{j r}\right) \geq 0$. When $x_{r j}=1$ or $x_{j r}=1$, these constraints are trivially valid. When $x_{r j}=0$ and $x_{j r}=0,2 \leq u_{j} \leq(n-2)$ and hence these constraints are valid.

Linearization phase: Linearize ATSP-MTZ1 and the constraints generated R1 through R4 by using the substitution $y_{i j}=u_{i} x_{i j}$ and $z_{i j}=u_{j} x_{i j}$ for $i, j \neq r, i \neq j$ and as in (11) and (12), by replacing $u_{j} x_{r j}$ by $x_{r j}$ and $u_{j} x_{j r}$ by $(n-1) x_{j r}$ for $j \neq r . z_{i j}$ can be eliminated through the relationship $z_{i j}=y_{i j}+x_{i j}, i, j \neq r, i \neq j$. The resulting formulation is ATSP-SD.

\section{Model ATSP-SD: ATSP Formulation of Sherali and Driscoll [18]}

In addition to (1)-(3) and (8)-(9)

$\sum_{(i, j) \in A, j \neq r} y_{i j}+(n-1) x_{i, r}=u_{i}, \quad i \neq r$

$\sum_{(i, j) \in A, i \neq r} y_{i j}+1=u_{j}, \quad j \neq r$

$x_{i j} \leq y_{i j} \quad(i, j) \in A, i, j \neq r$

$y_{i j} \leq(n-2) x_{i j} \quad(i, j) \in A, i, j \neq r$

$u_{j}+(n-2) x_{i j}-(n-1)\left(1-x_{j i}\right) \leq y_{i j}+y_{j i} \quad(i, j) \in A, i, j \neq r$

$y_{i j}+y_{j i} \leq u_{j}-\left(1-x_{j i}\right) \quad(i, j) \in A, i, j \neq r$

$1+\left(1-x_{r j}\right)+(n-3) x_{j r} \leq u_{j}, \quad j \neq r$

$u_{j} \leq(n-1)-(n-3) x_{r j}-\left(1-x_{j r}\right), \quad j \neq r$

$y_{i j} \geq 0 \quad(i, j) \in A$

In ATSP-SD, in addition to the variables $x_{i j}$ and $u_{i}$, the variable $y_{i j}$ that represents the rank-order of the $\operatorname{arc}(i, j)$ in the tour is used. The rank-order of the first arc in the tour, i.e., the arc originating at $r$, is zero while the rank-order of the last arc in the tour, i.e., the arc entering node $r$, is $n-2$. Each arc is assigned a unique value such that $y_{i j}=u_{i}$ or $y_{i j}=u_{j}-1$ given that $x_{i j}=1$. So, constraints can be interpreted accordingly by assigning appropriate values to variables $x_{i j}$. In this sense, constraints (13) and (14) are valid because there can be only one arc incoming to (outgoing from) a node. Constraints (15) and (16) are constraints coupling variables $x_{i j}$ and $y_{i j}$ and describing appropriate lower and upper bounds on $y_{i j}$. For constraints (17) and (18), when $x_{i j}=1$ (and hence $x_{j i}=0$ ), $y_{i j}+y_{j i}=\left(u_{j}-1\right)$. Because $y_{j i}=0$ and $y_{i j}=u_{i}$ as implied by constraints (16) and (13), respectively, $u_{i}=u_{j}-1$. In other words, constraints (17) and (18) require that the difference between $u_{i}$ and $u_{j}$ be exactly 1 when edge $\{i, j\}$ is in the solution. Constraints (19) and (20) require that $u_{j}=1$ when $x_{r j}=1$ and $x_{j r}=0$ and that $u_{j}=n-1$ when $x_{j r}=1$ and $x_{r j}=0$. Constraints (21) are non-negativity constraints for $y_{i j}$. 


\section{Adaptation of ATSP-SD formulation to HMST}

HMST is formulated on a directed network $G^{\prime}=(V, A)$ obtained from $G=(V, E)$ by replacing each undirected edge $\{i, j\} \in E$ by two directed $\operatorname{arcs}(i, j)$ and $(j, i)$ with symmetric costs $c_{i j}=c_{j i}$. A feasible solution to HMST is an arborescence (e.g., [1]), i.e., a directed tree such that every node other than the root node has exactly one incoming arc while the root node has no incoming arc.

In this section, two models are developed for HMST. The first one is the basic model HMST-MTZ. It is basic in the sense that it is based on the original MTZ constraints [12] and that no liftings or extensions to MTZ constraints are used. For extensions and improvements to HMST-MTZ, the reader may refer to Gouveia [9] and Akgün and Tansel [20]. The second model is HMST-SD obtained by adapting SD constraints [18] to formulate HMST.

\section{Model HMST/MTZ: HMST formulation based on basic MTZ constraints}

In addition to (1), (5)-(6), and (8)-(9),

$\sum_{i} x_{i j}=1, \quad j \in(V-r)$

$u_{i}-u_{j}+n x_{i j} \leq n-1 \quad(i, j) \in A, j \neq r$

$u_{i} \leq p, \quad i \in(V-r)$

In the context of HMST, constraints (23) prevent subtours by assigning labels in such a way that each directed arc included in the arborescence is directed from a node with a lower label into a node with a higher label. This ensures that the node labels form an increasing sequence on any directed path so that any node previously visited on a directed path cannot be re-visited, thereby preventing formation of subtours. The uniqueness of node labels is not required. This actually allows feasible solutions with different labeling structures. Specifically, in the assignment of labels to nodes, there are three possible cases for an edge $\{i, j\}$ : either $x_{i j}=1$, or $x_{j i}=1$, or both $x_{i j}=0$ and $x_{j i}=0$. If $x_{i j}=1$, then $u_{j} \geq u_{i}+1$. Similarly, if $x_{j i}=1$, then $u_{i} \geq u_{j}+1$. If both $x_{i j}=0$ and $x_{j i}=0$, then $u_{i}-u_{j} \leq n-1$ and $u_{j}-u_{i} \leq n-1$. The upper bounds on the labels defined by constraints (24) eliminate the paths with more than $p$ arcs satisfying the hop requirement. This can also be achieved by changing the constraints (23) as $u_{i}-u_{j}+p x_{i j} \leq p-1$ [9], which is based on the fact that the upper bound on the value of $u_{i}$ is not $n-1$ but $p$ in HMST. When this set of constraints is used instead of (23), the resulting model is stronger than HMST-MTZ [9].

Constraints (22) require that the number of incoming arcs to any non-root node be equal to 1 . Unlike constraints (3), constraints (22) are not defined for $i=r$ because no arc is allowed to enter node $r$. Constraints (22) together with (23) establish that the resulting tree is directed from the root.

Note that constraints (23) are slightly different from constraints (4). Constraints (4) are defined for arcs $(i, j)$ with $i, j \neq r$ while constraints (23) are defined for arcs $(i, j)$ with $j \neq r$. Accordingly, because $p \leq n-1$ is assumed, bounds in constraints (23) are changed to allow $u_{r}-u_{j} \leq n-1$ for $j \neq r$. Constraints (23) can be split into two, one set for $\operatorname{arcs}(r j)$ with $j \neq r$ and one set for $\operatorname{arcs}(i, j)$ with $i, j \neq r$. In this case, constraints (23) and (4) can be used for the former and latter arc sets, respectively. However, the given form is preferred to be consistent with the previous studies.

\section{Model HMST/SD: HMST formulation based on SD constraints}

In addition to (1), (5), (6), (8), (9), (14), (15), (21), (22), and (24),

$\sum_{(i, j) \in A, j \neq r} y_{i j}+p \geq u_{i}, \quad i \in V$ $y_{i j} \leq(p-1) x_{i j} \quad(i, j) \in A, j \neq r$

$u_{j}+(p-1) x_{i j}-p\left(1-x_{j i}\right) \leq y_{i j}+y_{j i} \quad(i, j) \in A, i, j \neq r$

$y_{i j}+y_{j i} \leq u_{j}-\left(1-x_{j i}\right) \quad(i, j) \in A, i, j \neq r$

$2-x_{r j} \leq u_{j}, \quad j \neq r$

$u_{j} \leq p-(p-1) x_{r j}, \quad j \neq r$

Note that the upper bounds on the values of $u_{i}$ and $y_{i j}$ are $p$ and $p-1$ in HMST, respectively. Hence, the bounds/coefficients in all constraints are changed accordingly. In essence, the bounds/ coefficients of $n-1$ and $n-2$ are changed to $p$ and $p-1$, respectively. Other constraint-specific changes are discussed next. Constraints (25) replace constraints (13). In a feasible solution to HMST, there may be no arcs or more than one arc leaving a node $i$ and hence constraints (13) are not valid. If there is more than one arc outgoing from a node $i$, then $\sum_{(i, j) \in A, j \neq r} y_{i j} \geq u_{i}$ holds. If there is no outgoing arc from $i$, i.e., $i$ is a leaf node, then $\sum_{j \neq r} y_{i j}=0$ and constraint (13) becomes infeasible because $u_{i}>0$. However, it can be made feasible by adding the highest possible node label value $p$ to the left-hand side of the constraint. Constraints (14) are valid because only one incoming arc to a node is allowed. During computational studies, these constraints are used in $\leq$ form because better solution times are obtained with this form. Of the coupling constraints (15) and (16), constraints (15) are valid and hence used as is while constraints (16) are replaced by constraints (26). Constraints (26) are similar to constraints (16); however, the upper bound on the variable $y_{i j}$ is now $p-1$ because the highest possible label value of a non-root node is $p$. Constraints (27) and (28) are the same as constraints (17) and (18) but with coefficients adapted according to the bounds on $y_{i j}$ and $u_{i}$. Constraints (19) and (20) are not valid for HMST because an arc of the form $(j, r)$ is not allowed. However, the information provided for the label value of a node $j \neq r$ depending on whether it is directly connected to $r$ or not can be extracted and incorporated as given by constraints (29) and (30). Constraints (29) and (30) require that the value of $u_{j}$ be equal to 1 if $x_{r j}=1$, i.e., node $j$ is incident to the root node, and to be greater than 2 if $x_{r j}=0$, i.e., node $j$ is not incident to the root node. These constraints are actually liftings to constraints (6) and (24) offered in Gouveia [9]. Note that the formulation is valid without constraints (5), (6) and (24) as well. However, they are included in the model to restrict the values of the variables $u_{i}$. Notice that the formulation is still valid without making the changes in the constraints regarding the bounds on the values of $u_{i}$ and $y_{i j}$ as long as constraints (24) are in the formulation.

Computational studies show that HMST-SD is in general better than the previous MTZ-based models with respect to LP bounds; however, there are few cases where the LP bounds of HMST-SD are inferior to those of some previous models. This has led to seeking for new ways of strengthening HMST-SD. Because the set of topological and MTZ-based constraints offered by Akgün and Tansel [20] have contributed to increase the LP bounds of the models significantly, it has been decided to incorporate them into HMST-SD. The following section describes those constraints and how the formulation is adapted to the new constraints. Computational studies show that the improved formulations give better LP bounds than all previous MTZ-based models for HMST.

\section{Improved formulations for HMST}

Akgün and Tansel [20] offer two sets of constraints for HMST, one is topological and the other is MTZ-based. The first set consists of topology-enforcing constraints in the sense that it tries 
to define the topology of a feasible solution to HMST with some additional features. The second is MTZ-based in the sense that it allows to select a feasible solution with a certain labeling structure. In the following, these sets of constraints are defined briefly and then incorporated into HMST/SD. The reader is referred to Akgün and Tansel [20] for details of the constraints.

\subsection{Topological constraints}

HMST-SD is structured around expressing the assignment of node and arc labels as exactly as possible. However, a feasible solution to HMST has some structural implications that can be used to further improve HMST-SD. In a feasible solution to HMST, a node is implicitly assigned to be either a leaf node or a central (non-leaf) node. If the degree of a node is 1 , then that node is a leaf. Otherwise, it is a central node. If the root node is a leaf node, it has one outgoing arc but no entering arcs. Any non-root node that is a leaf node has one incoming arc but no outgoing arcs. If the root node is a central node, it has 2 or more outgoing arcs but no incoming arcs while a non-root node that is a central node has one incoming arc and at least 1 outgoing arc. This structure allows the number of inward and outward arcs of a node to be determined depending on its type or vice versa. Similarly, the connectivity between two nodes can be specified by their types, e.g., two leaf nodes cannot be linked in a feasible solution. That the resulting solution is a tree implies that only a single arc in one direction is possible. The aforementioned structural/topological properties allow the following set of constraints, ITEF, to be developed.

Let $w_{i c}$ and $w_{i l}$ be a pair of binary variables associated with node $i$ with $w_{i c}=1\left(w_{i l}=1\right)$ if node $i$ is a central (leaf) node and $w_{i c}=0\left(w_{i l}=0\right)$ if not.

ITEF: Improved set of topology-enforcing constraints

In addition to constraints (24),

$w_{i c}+w_{i l}=1, \quad i \in V$

$\sum_{j} x_{i j} \geq 1, \quad i=r$

$\sum_{j \neq r} x_{i j} \geq 1+w_{i c}, \quad i=r$

$\sum_{j \neq r} x_{i j} \leq(n-1)-(n-2) w_{i l}, \quad i=r$

$\sum_{j} x_{j i}+\sum_{j \neq r} x_{i j} \geq 1+w_{i c}, \quad i \in(V-r)$

$\sum_{j} x_{j i}+\sum_{j \neq r} x_{i j} \leq(n-1)-(n-2) w_{i l}, \quad i \in(V-r)$

$\sum_{j \neq r} x_{i j} \geq 1-w_{i l}, \quad i \in(V-r)$

$x_{i j} \leq w_{i c} \quad(i, j) \in A, i, j \neq r$

$x_{i j}+w_{i l}+w_{j l} \leq 2 \quad(i, j) \in A, j \neq r$

$x_{i j} \equiv 0 \quad(i, j) \in A, j=r$

$x_{i j}+x_{j i} \leq 1 \quad(i, j) \in A, i<j$

$\sum_{j \neq i} x_{i j}=n-1$

$w_{i c}, w_{i l} \in\{0,1\}, \quad i \in V$
Note that constraints (31)-(43) address topological requirements other than the hop requirement. Therefore, constraints (31)-(43) are a valid topology-enforcing formulation for HMST only when combined with a set of constraints that imposes the hop requirement, e.g., (24) as given.

Constraints (31) require that each node be either a leaf node or a central node. Constraints (32) through (42) express some structural properties of a feasible solution. Constraints (32) through (34) define lower and upper bounds on the number of outgoing arcs from the root node. Constraint (32) establishes that the number of outgoing arcs at the root node $r$ is at least 1 . Constraints (33) and (34) require that the number of outgoing arcs at the root node be equal to 1 when $r$ is a leaf node and be at least 2 and at most $n-1$ when node $r$ is a central node.

Constraints (35) through (37) set upper and lower limits on the degree of non-root nodes. Constraints (35) state that the total number of outward and inward arcs of each non-root node is at least 2 when a non-root node is a central node and at least 1 when a non-root node is a leaf node. Constraints (36) restrict the number of inward and outward arcs of a non-root node to be at most 1 when the node is a leaf node and at most $n-1$ when the node is a central node. Constraints (36) together with constraints (22) ensure that the number of inward (outward) arcs of a nonroot node is $1(0)$ when the node is leaf node and 1 (greater than 1 ) when the node is a central node. Constraints (37) require that the number of outward arcs of a non-root node is greater than 1 (0) when the node is a central (leaf) node.

Constraints (38) require that a non-root node be a central node if there is an outgoing arc from it. Constraints (39) prevent arcs between pairs of leaf nodes. Constraints (40) do not allow any arcs incoming to the root node. Constraints (41) state that a pair of arcs of opposite directions between a pair of nodes is not possible. Constraints (42) require that the total number of arcs in the solution be equal to $n-1$, which is a known fact for a tree (e.g., [1]). Finally, constraints (43) give the zero/one restrictions on the decision variables $w_{i c}$ and $w_{i l}$.

Akgün and Tansel [39] use a similar set of constraints as degree-enforcing constraints in the context of the MinimumDegree Constrained MST, where a minimum-cost spanning tree is sought for while requiring that each node in the tree be either a leaf node or a central node that is adjacent to at least $d$ nodes. The results show that the proposed models give significantly better solution times than the ones in the literature based on single- and multi-commodity flow models.

\subsection{MTZ-based constraints}

In a feasible solution of HMST, each node is either a central node or a leaf node. Because a non-root leaf node has one incoming arc whose origin is necessarily a central node, then a feasible solution can be obtained by requiring that the labels of all non-root leaf nodes be greater than the highest possible label of central nodes. This condition is easily fulfilled if we assign the label value $p$ to each non-root leaf node while permitting central nodes to take label values of at most $p-1$. In this case, if all nodes other than the root node are leaf nodes, then the root node receives the node label of 0 and all other nodes receive node labels of $p$. If there is a non-root central node, then its label will be between 1 and $p-1$. Thus, in finding feasible solutions for HMST, looking only for solutions in which the label values of nonroot leaf nodes are restricted to $p$ and the label values of central nodes are restricted to be less than or equal to $p-1$ is sufficient. This can be achieved by adding the following constraints:

$u_{i} \geq p w_{i l}, \quad i \in(V-r)$

$u_{i} \leq p-w_{i c}, \quad i \in(V-r)$ 
Constraints (44) together with an appropriate hop constraint require that the labels of all non-root leaf nodes be equal to $p$. Constraints (45) restrict the labels of central nodes to be at most $p-1$.

\subsection{Improved formulations for HMST}

In this section, two improved formulations for HMST are given. The first formulation is obtained by combining directly HMST-SD with ITEF without any changes in the constraints. The second formulation is obtained by combining HMST-SD, ITEF, and MTZ-based constraints with some changes in the constraints of HMST-SD.

Model HMST-SD1: The first improvement to HMST-SD

(1), (5), (6), (8), (9), (14), (21), (22), and (24)-(43)

A feasible solution for HMST-SD can be obtained from a feasible solution of HMST-SD1 by treating the variables $w_{i l}$ and $w_{i c}$ as nonexistent and using only the values of the remaining variables. In this regard, HMST-SD1 is different from HMST-SD with only additional information provided about whether nodes are leaf or central. Clearly, the same information can actually be derived without using the values of the variables $w_{i l}$ and $w_{i c}$ by the degree of the nodes. However, additional constraints to HMST-SD improves the LP bounds such that the LP bounds of HMST-SD1 are greater than or equal to the LP bounds of all previous MTZ-based models. However, the next model HMST-SD2 gives better LP bounds than HMST-SD1.

Model HMST-SD2: The second improvement to HMST-SD

In addition to (1), (5)-(6), (8)-(9), (14), (21)-(22), (24)-(27), (31)-(43), and (44)-(45)

$y_{i j} \leq u_{j}-\left(1-x_{j i}\right) \quad(i, j) \in A, i, j \neq r$

$u_{j} \geq 2-x_{i j}+(p-2) w_{j l}, \quad i=r, j \in(V-r)$

$u_{j} \leq p-(p-1) x_{i j}+(p-1) w_{j l}, \quad i=r, j \in(V-r)$

HMST-SD2 is different from previous models in the sense that it allows feasible solutions only with a certain node and arc labeling structure. In this regard, a feasible solution to HMST-SD2 is not necessarily feasible to the previous models discussed in this paper or vice versa. Specifically, in a feasible solution to HMSTSD2, $u_{j}=p$ and $y_{i j}=p-1$ when $x_{i j}=1$ for a leaf node $j$. Clearly, $u_{j}=p$ is required by constraints (44) and (24). Given that $u_{j}=p, y_{i j}=p-1$ is required by constraints (27) because $y_{i j} \geq u_{j}-1$ when $x_{i j}=1$. In a feasible solution to HMST-SD and HMST-SD1, the values for $u_{i}$ and $y_{i j}$ give the true distance of node $i$ and arc $(i, j)$ from the root, respectively. On the other hand, for HMST-SD2, they do not necessarily give the true distance. For example, for $p \geq 2$, the node label of a leaf node directly connected to the root is $p$ even though its true distance is 1.

Constraints (46) in HMST-SD2 are used instead of constraints (28) because when constraints (28) are used with constraints (44), the solution obtained may not necessarily be optimal for the original problem. Recall that constraints (17) and (18) and hence constraints $(27)-(28)$ require that the difference between $u_{i}$ and $u_{j}$ be exactly 1 when edge $\{i, j\}$ is in the solution (because $u_{j}-1 \leq y_{i j} \leq u_{j}-1$ and $u_{j} \leq y_{j i} \leq u_{j}$ when $x_{i j}=1$ and $x_{j i}=1$, respectively). Thus, when constraints (28) are used with constraints (44), the following situation occurs. For each leaf node $j$ and for some $i, x_{i j}=1$ and hence $y_{i j}=p-1$ is required because $u_{j}=p$. However, this requires that, for some $k \neq i, x_{k i}=1$ and hence $y_{k i}=p-2$. This goes in a similar manner until the root node $r$ is reached. What happens actually when constraints (28) are used with constraints (44) is that HMST-SD2 produces only solutions in which there is a path from the root to each leaf node whose length is exactly $p$, i.e., the distance between the root and each leaf node is $p$. There may be cases where such a solution is desirable. If this is the case, then HMST-SD2 with constraints (46) replaced by constraints (28) can be used. On the other hand, if there is no such a requirement, such a solution may not be optimal for the original problem as the solutions in which leaf nodes are at different distances from the root are disregarded. To eliminate such a possibility, we need to get rid of the requirement that the difference between $u_{i}$ and $u_{j}$ be exactly 1 when edge $i, j$ is in the solution. This can be done in several ways. We achieve this by constraints (46). Note that these constraints only require that $y_{j i} \leq u_{i}-1$ when $x_{j i}=1$. Thus, $y_{j i}$ does not have to be equal to $u_{j}$ as required by $u_{j} \leq y_{j i} \leq u_{j}$ that results from constraints (27) and (28) for $x_{j i}=1$. Another option to use instead of constraints (46) is to add $p$ to the right-hand side of constraints (28).

Constraints (47) and (48) replace constraints (29) and (30), respectively, to adapt to the requirement imposed by constraints (44). Consider a solution in which the root $r$ is a central node and leaf node(s) $i$ is directly connected to the root. Constraints (30) require node $i$ to take on a label value of 1 while constraints (44) require node $i$ to take on a label value of $p$. However, this is not feasible and hence an optimal solution with the aforementioned property is not possible as such solutions are excluded from the feasible region. To correct this situation and to be consistent with constraints (44), constraints (29) and (30) are changed as given by constraints (47) and (48). Constraints (47) establish that the value of $u_{j}$ is greater than or equal to 2 if node $j$ is a central node and $x_{r j}=0$ and that the value of $u_{j}$ is greater than or equal to $p$ if node $j$ is a leaf node and $x_{r j}=0$. Constraints (48) require that the value of $u_{j}$ be equal to 1 if node $j$ is a central node and $x_{r j}=1$ and that the value of $u_{j}$ be less than or equal to $p$ if node $j$ is a leaf node and $x_{r j}=1$. In all other cases, constraints (47) and (48) become redundant.

\subsection{MTZ-based models in the literature used to compare the proposed models}

The proposed models HMST/SD, HMST/SD1, and HMST/SD2 are compared with two models, I/E8I and Rel-M [20]. I/E8I is the model that gives the best LP bounds while Rel-M is the model that gives best solution times among the the previous MTZ-based models. The aformentioned models are given next.

Model I/E8I (48)

In addition to (1), (5), (6), (8), (22), (31)-(43), (45), (47), and

$(p-2) x_{j i}+u_{i}-u_{j}+p x_{i j} \leq p-1 \quad(i, j) \in A, j \neq r$

$\sum_{k=1, k \neq i} x_{k j}+(p-3) x_{j i}+u_{i}-u_{j}+p x_{i j} \leq p-1 \quad(i, j) \in A, j \neq r, p \geq 3$

Constraints (49) and (50) are the liftings to constraints (23) offered by Gouveia [9]. Constraints (49) require the values of variables $u_{i}$ and $u_{j}$ to differ exactly by 1 if edge $\{i, j\}$ is in the solution, i.e., whenever $x_{i j}=1$ or $x_{j i}=1$. Note that $x_{i j}=0$ when $\sum_{k \neq i, r} x_{k j}=1$ due to constraints (22) and hence node $j$ cannot be adjacent to node $r$ when this occurs. Thus, the difference between the values of $u_{i}$ and $u_{j}$ is at most $p-2$. This result and the fact that $x_{i j}+x_{j i} \leq 1$ can be combined to obtain liftings (50) valid for $p \geq 3$. Notice that because constraints (49) and (50) require $u_{i}$ and $u_{j}$ to differ exactly by one if edge $\{i, j\}$ is in the solution, using constraints (44) with constraints (49) and (50) may cause infeasibility. Hence, constraints (44) are not used in I/E8I. 
Model Rel-M

In addition to (1), (5), (6), (8), (22), (23), (24), (31)-(34), (36), (38)-(43), (44), (45), and (47)

$\sum_{j \neq r} x_{i j} \geq w_{i l}-1, \quad i \in(V-r)$

Rel-M does not include constraints (35) and (37). The exclusion of constraints from Rel-M permits the variables $w_{i c}$ and $w_{i l}$ to take on values of 1 and 0 , respectively, when a nonroot node $i$ is in fact a leaf node. Even though this does not agree with the intended meaning attached to these variables, such solutions define feasible trees. A more liberal interpretation of the auxiliary variables $w_{i c}$ and $w_{i l}$ in Rel-M is taken. They are interpreted as node labels that generally distinguish central nodes from leaf nodes but sometimes with incorrect values.

Note that the left sides of constraints (37) and (51) are the same and specify the total number of outgoing arcs at a non-root node while the right sides of (37) and (51) are the negatives of each other $\left(1-w_{i l}\right.$ and $\left.w_{i l}-1\right)$. Accordingly, constraints (51) may be considered as a replacement for (37). However, the use of (51) in place of (37) in the proposed models is not recommended as its use in these models generally worsens the solution times while its use in Rel-M improves the solution times.

\section{Computational tests}

Computational studies are performed by using specially structured test problems from the literature (e.g., [26]). Test problems consist of three 20 -node, three 40 -node, three 60 -node, and one 80-node complete networks with 210,820,1830, and 3240 edges, respectively. For the first three network sizes, two Euclidean instances, TC and TE, and one random instance, TR, are considered. For 80-node network, only TE instances are considered. Euclidean instances differ from each other based on the location of the root node. In TC instances, the root is located in the center of the grid while in TE instances the root is located on a corner of a grid. For each instance, hop parameter values are set to $3-5$.

The size of each instance is reduced by applying an arcelimination test used in the literature (e.g., $[21,26])$. The test is based on the fact that if $c_{i j}>c_{r j}$, then any optimal solution does not use arc $(i, j)$ and if $c_{i j}=c_{r j}(i \neq r)$, then there is an optimal solution without arc $(i, j)$. Thus, arc $(i, j)$ can be eliminated whenever $c_{i j} \geq c_{r j}$. The number of arcs remaining after the elimination test in 20-node, 40-node, and 60-node networks for TC, TE, and TR instances are $38 \%, 82 \%$, and $52 \%, 33 \%$, $75 \%$, and $51 \%$, and $31 \%, 74 \%$, and $58 \%$ of the original numbers, respectively.

Computational tests are performed on a PC with a $3.0 \mathrm{GHz}$ Intel Core 2 Duo processor and 3 GB of RAM by using ILOG CPLEX 9.0. The models are run until optimality is attained or for $10 \mathrm{~h}$ (36,000 CPU seconds) at maximum and by using default settings of CPLEX (e.g., moving the best bound strategy for branching is used, cuts are allowed) except that file storage is set to 3 , which allows tree file to be stored on the hard disk when it reaches the default limit in order not to run out of memory (ILOG CPLEX [40]).

In the tables presenting computational studies, LP relaxation bounds, run times, optimal objective function values, and relative optimality gaps are given. The relative optimality gap is defined as $|\mathrm{BP}-\mathrm{BF}| /\left(1^{-10}+|\mathrm{BP}|\right)$, where $\mathrm{BP}$ is the objective function value of the best integer solution and $\mathrm{BF}$ is the best remaining objective function value of any unexplored node [40].

\subsection{LP relaxation bounds}

Table 1 gives LP relaxation bounds for HMST/MTZ, I/E8I, HMST-SD, HMST/SD1, and HMST/SD2. I/E8I gives better LP bounds than the basic model HMST/MTZ for all 27 instances. The improvements over the basic model change from 3.6\% (Pr.Id. 1) to $22.4 \%$ (Pr.Id. 6) with an average of $9.4 \%$. On the average, the largest and the smallest improvements are 20.5\% and 3.9\% for TE 20 and TC 60 instances, respectively. The average improvements for TC, TE, and TR instances are 5.3\%, 12.1\%, and $10.6 \%$, respectively.

The LP bounds of HMST/SD are greater than or equal to the LP bounds of I/E8I for 25 instances out of 27 except for Pr. Id. 6 and Pr. Id. 15, both of which are TE instances. For one instance (Pr. Id. 9), the LP bounds of the models are the same. The increases in the LP bounds for the remaining 24 instances change from $0.08 \%$ to $9.8 \%$ with an average of $3.53 \%$. For Pr.Id. 6 and 15 , the LP bounds of HMST/SD are $2 \%$ and $0.5 \%$ less than those of I/E8I, respectively. On the average, the LP bounds of HMST/SD are $3 \%$ better than those of I/E8I. The highest improvements are obtained for instances with $p=3$. As the value of $p$ decreases, the increase in the LP bounds decreases. Note that for both Pr. Id. 6 and Pr.Id. 15, $p=5$. The average improvements are $3 \%, 2.5 \%$, and $3.6 \%$ for TC, TE, and TR instances, respectively.

The LP bounds of HMST/SD1 are greater than or equal to the LP bounds of both HMST/SD and I/E8I. HMST/SD1 and HMST/SD have

Table 1

LP relaxation bounds for the models.

\begin{tabular}{|c|c|c|c|c|c|c|c|}
\hline Pr. Id. & Pr. type & $p$ & HMST/MTZ & I/E8I & HMST/SD & HMST/SD1 & HMST/SD2 \\
\hline 1 & TC 20 & 3 & 294.67 & 307.00 & 319.69 & 319.69 & 320.95 \\
\hline 2 & & 4 & 290.00 & 303.71 & 304.89 & 304.89 & 306.00 \\
\hline 3 & & 5 & 289.20 & 302.75 & 303.00 & 303.00 & 303.00 \\
\hline 4 & TE 20 & 3 & 258.67 & 303.67 & 328.77 & 336.87 & 339.93 \\
\hline 5 & & 4 & 248.00 & 301.80 & 302.45 & 314.88 & 314.93 \\
\hline 6 & & 5 & 246.00 & 301.00 & 295.05 & 310.57 & 310.57 \\
\hline 7 & TR 20 & 3 & 128.67 & 140.70 & 148.00 & 148.00 & 151.83 \\
\hline 8 & & 4 & 124.50 & 138.33 & 139.67 & 139.67 & 139.67 \\
\hline 9 & & 5 & 122.40 & 137.00 & 137.00 & 137.00 & 137.00 \\
\hline 10 & TC 40 & 3 & 446.00 & 472.67 & 518.07 & 518.07 & 525.29 \\
\hline 11 & & 4 & 437.00 & 472.00 & 483.32 & 483.32 & 484.76 \\
\hline 12 & & 5 & 435.20 & 472.00 & 476.28 & 476.28 & 476.99 \\
\hline 13 & TE 40 & 3 & 458.67 & 489.86 & 519.35 & 520.48 & 528.89 \\
\hline 14 & & 4 & 456.00 & 487.83 & 490.48 & 494.28 & 494.55 \\
\hline 15 & & 5 & 455.60 & 487.02 & 484.43 & 490.10 & 490.10 \\
\hline 16 & TR 40 & 3 & 117.33 & 129.80 & 142.53 & 142.53 & 144.49 \\
\hline 17 & & 4 & 114.50 & 127.16 & 131.14 & 131.14 & 131.25 \\
\hline 18 & & 5 & 113.40 & 126.50 & 128.29 & 128.29 & 128.42 \\
\hline 19 & TC 60 & 3 & 641.33 & 664.73 & 713.17 & 713.17 & 725.93 \\
\hline 20 & & 4 & 635.00 & 659.33 & 671.16 & 671.16 & 673.50 \\
\hline 21 & & 5 & 632.80 & 658.92 & 663.17 & 663.17 & 663.45 \\
\hline 22 & TE 60 & 3 & 896.67 & 968.80 & 1036.47 & 1036.47 & 1047.41 \\
\hline 23 & & 4 & 873.00 & 953.92 & 973.56 & 973.56 & 974.21 \\
\hline 24 & & 5 & 866.00 & 951.04 & 960.78 & 960.78 & 961.47 \\
\hline 25 & TR 60 & 3 & 162.00 & 181.08 & 198.19 & 198.19 & 201.16 \\
\hline 26 & & 4 & 158.75 & 173.39 & 176.94 & 176.94 & 177.22 \\
\hline 27 & & 5 & 157.80 & 172.08 & 172.79 & 172.79 & 172.88 \\
\hline
\end{tabular}


the same LP bounds for 21 instances out of 27 . In the remaining six instances, all of which are TE instances, the improvements in the LP bounds change from $0.2 \%$ to $5.3 \%$ with an average of $2.3 \%$. On the average, the improvement over all instances is $0.52 \%$. This result is expected because HMST/SD1 is composed of improved topology enforcing (ITEF) constraints defined in Akgun and Tansel [20] and SD constraints while HMST/SD is composed of only SD constraints. Thus, adding ITEF constraints to HMST/SD strengthens the formulation.

HMST/SD1 gives better LP bounds than I/E8I for all instances except Pr. Id. 9 for which both models have the same LP bounds. The increases in the LP bounds for 26 instances change from $0.08 \%$ to $10.93 \%$ with an average of $3.7 \%$. On the average, the improvement over all instances is $3.57 \%$. On the average, the lowest and highest improvements are $1.5 \%$ and $6.15 \%$ for TC 20 and TE 20 instances, respectively. Average improvements are 3\%, $4.08 \%$, and $3.6 \%$ for TC, TE, and TR instances, respectively. This result is also expected because I/E8I is composed of ITEF constraints and some liftings to MTZ constraints based on Desrochers and Laporte [16] that are dominated by SD constraints while HMST/SD1 is composed of ITEF and SD constraints. Note that even though HMST/SD is not stronger than I/E8I, it is stronger than models offered by Gouveia [9] which do not include ITEF but the aforementioned liftings to MTZ constraints.

HMST/SD2 gives the best LP bounds among all MTZ-based models in the literature and in this paper. HMST/SD2 and HMST/ SD1 have the same LP bounds for five instances out of 27. For the remaining 22 instances, the improvements in the LP bounds change from $0.01 \%$ to $2.59 \%$ with an average of $0.66 \%$. The average improvement over all instances is $0.53 \%$. The average improvements for TC, TE, and TR instances are $0.53 \%, 0.42 \%$, and $0.65 \%$, respectively.

HMST/SD2 gives better LP bounds than HMST/SD for 24 instances while both models have the same LP bounds for three instances. The increases in the LP bounds for 24 instances change from $0.04 \%$ to $5.3 \%$ with an average of $1.19 \%$. The average overall improvement is $1.05 \%$ while the average improvements for TC, TE, and TR instances are $0.53 \%, 2 \%$, and $0.65 \%$, respectively. HMST/SD2 gives better LP bounds than I/E8I for all instances except for Pr. Id. 9 for which the LP bounds are the same. For 26 instances, the improvements in the LP bounds change from $0.08 \%$ to $11.94 \%$ with an average of $4.3 \%$. The average overall improvement is $4.14 \%$ while the average improvements for TC, TE, and TR instances are 3.59\%, $4.53 \%$, and $4.3 \%$, respectively. HMST/SD2 improves the LP bounds of HMST/MTZ from $4.8 \%$ to $31.41 \%$ with an average of $13.91 \%$.

The results in Table 1 indicate that using only the SD constraints to formulate HMST does not produce a model stronger than the previous models in the literature. When HMST/SD is combined with ITEF, a set of constraints proposed by Akgün and Tansel [20], the resulting model HMST/SD1 gives better LP bounds than all previous MTZ-based models. However, HMST/SD2 composed of modified SD constraints and ITEF constraints together with a new set of MTZ-based constraints offered by Akgün and Tansel [20] is stronger than all models in the literature and in this paper.

\subsection{Solution times}

Table 2 gives solution times, best objective function values, and relative optimality gaps for Rel-M, HMST/SD, and HMST/SD2. The results for HMST/SD1 are not given because the solution times for HMST/SD1 are significantly worse than those of HMST/ SD2.

The results show that all TC 20, TE 20, TR 20, TC 40, and TR 40 instances are solved to optimality by all three models. TR 60 instances are solved optimally by Rel-M and HMST/SD2 while one instance (Pr. Id. 25) cannot be solved by HMST/SD. No optimal solutions are obtained for other instances in the allotted time.

For TC 20 and TR 20 instances, the solution times are less than $1 \mathrm{~s}$. For TE 20 instances, the solution times change from $68.13 \mathrm{~s}$ (HMST/SD2) to $2557 \mathrm{~s}$ (HMST/SD). The average solution times of 117,1434 , and $490 \mathrm{~s}$ are obtained for Rel-M, HMST/SD, and HMST/ SD2, respectively. For TC 40 instances, the solution times change from $196 \mathrm{~s}$ (Rel-M) to 22,887 s (HMST/SD). The average solution times are $803,11,734$, and $7527 \mathrm{~s}$ for Rel-M, HMST/SD, and HMST/ SD2, respectively. For TR 40 instances, the average solution times are $6.5,25.5$, and $34.4 \mathrm{~s}$ for Rel-M, HMST/SD, and HMST/SD2, respectively. The best and worst solution times are $2.01 \mathrm{~s}$ (Rel-M) and $68.58 \mathrm{~s}$ (HMST/SD2). For TR 60 instances, the solution times change from 45.26 to $17,228 \mathrm{~s}$ for the problems solved to optimality. The average solution times for Rel-M and HMST/SD2 are 1565 and 2079 s, respectively.

For TE 40 instances, the average optimality gaps for Rel-M, HMST/SD, and HMST/SD2 are $13.11 \%, 13.96 \%$, and $12.10 \%$, respectively. The best and worst gaps of $10.58 \%$ and $14.96 \%$ are obtained for HMST/SD2 and HMST/SD, respectively. For TC 60 instances, the average optimality gaps for Rel-M, HMST/SD, and HMST/SD2 are $7.62 \%, 7.17 \%$, and $6.38 \%$, respectively. The best and worst gaps of 5.63\% and 9.39\% are obtained for HMST/SD2 and Rel-M, respectively. For TE 60 instances, the average optimality gaps are $23.76 \%, 22.95 \%$, and $22.94 \%$, respectively. The best and worst gaps of $23.25 \%$ and $26.25 \%$ are obtained for HMST/SD2 and Rel-M, respectively. The figures imply that HMST/ SD2 is better than the other models with respect to reaching the optimality even though the difference is not significant. The lower bounds reached at the end of the allotted time are also compared to better assess Rel-M and HMST/SD2. The results show that the lower bounds of HMST/SD2 are higher than those of Rel-M implying that HMST/SD2 is likely to have better solution times than Rel-M for the problems not solved optimally.

The aforementioned results show that TE instances are much more difficult to solve than the two other sets of instances and that TC instances are harder to solve than TR instances. The results also show that Rel-M gives significantly better solution times than SD-based models offered in this paper with respect to the models solved to optimality. Even though the LP bounds of HMST/SD2 are better on the average about $4 \%$ than those of Rel$\mathrm{M}$, this dominance is not reflected in the solution times for those problems. On the other hand, the results for TE 40, TC 60, and TE 60 instances imply that HMST/SD2 is likely to give better solution times than Rel-M as the network size gets larger.

\subsection{Comparison of the proposed models to the flow-based and hop-indexed formulations}

HMST/SD2 is compared to the compact flow-based and hopindexed formulations of HMST by using the results given for the same test instances in Dahl et al. [26]. The results show that both flow-based and hop-indexed formulations give significantly better LP bounds than HMST/SD2. HMST/SD2 gives the same LP bound for only one instance (Pr. Id. 9) out of 27 instances. Specifically, the hop-indexed formulation, which produces better results than the flow-based formulation, is on the average $17 \%$ better than HMST/SD2 with the highest difference being $44 \%$ ( $\mathrm{Pr}$. Id. 22). The flow-based formulation, for which the results for 60node instances are not provided in Gouveia [26], is on the average $11 \%$ better than HMST/SD2 with the highest difference being $28 \%$ (Pr. Id. 13). In this regard, the LP bounds of the MTZ-based formulations still need to be improved significantly. 
Table 2

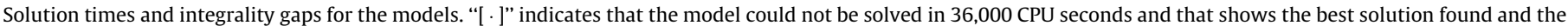
integrality gap at termination.

\begin{tabular}{|c|c|c|c|c|c|c|c|c|}
\hline \multirow[t]{2}{*}{ Pr. Id. } & \multirow[t]{2}{*}{ Pr. type } & \multirow[t]{2}{*}{$p$} & \multicolumn{2}{|l|}{ Rel-M } & \multicolumn{2}{|l|}{ HMST/SD } & \multicolumn{2}{|l|}{ HMST/SD2 } \\
\hline & & & [BP-Gap\%] & Time & [BP-Gap\%] & Time & [BP-Gap\%] & Time \\
\hline 1 & TC 20 & 3 & 340 & 0.15 & 340 & 0.17 & 340 & 0.34 \\
\hline 2 & & 4 & 318 & 0.11 & 318 & 0.08 & 318 & 0.11 \\
\hline 3 & & 5 & 312 & 0.04 & 312 & 0.08 & 312 & 0.08 \\
\hline 4 & TE 20 & 3 & 449 & 139 & 449 & 425 & 449 & 68.13 \\
\hline 5 & & 4 & 385 & 149 & 385 & 1319 & 385 & 990 \\
\hline 6 & & 5 & 366 & 62.6 & 366 & 2557 & 366 & 413 \\
\hline 7 & TR 20 & 3 & 168 & 0.06 & 168 & 0.02 & 168 & 0.03 \\
\hline 8 & & 4 & 146 & 0.05 & 146 & 0.03 & 146 & 0.05 \\
\hline 9 & & 5 & 137 & 0.01 & 137 & 0.02 & 137 & 0.02 \\
\hline 10 & TC 40 & 3 & 609 & 196 & 609 & 2172 & 609 & 2253 \\
\hline 11 & & 4 & 548 & 721 & 548 & 10,142 & 548 & 9537 \\
\hline 12 & & 5 & 522 & 1492 & 522 & 22,887 & 522 & 10,791 \\
\hline 13 & TE 40 & 3 & [713-13.18] & 36,000 & [712-14.96] & 36,000 & [708-10.58] & 36,000 \\
\hline 14 & & 4 & [627-13.39] & 36,000 & [627-14.14] & 36,000 & [627-13.50] & 36,000 \\
\hline 15 & & 5 & [594-12.76] & 36,000 & [599-12.78] & 36,000 & [595-12.21] & 36,000 \\
\hline 16 & TR 40 & 3 & 176 & 2.01 & 176 & 15.13 & 176 & 3.72 \\
\hline 17 & & 4 & 149 & 11.76 & 149 & 38.77 & 149 & 31.03 \\
\hline 18 & & 5 & 139 & 5.86 & 139 & 22.70 & 139 & 68.58 \\
\hline 19 & TC 60 & 3 & [866-6.64] & 36,000 & [866-6.54] & 36,000 & [866-6.80] & 36,000 \\
\hline 20 & & 4 & [792-9.39] & 36,000 & [783-8.10] & 36,000 & [782-6.70] & 36,000 \\
\hline 21 & & 5 & [734-6.84] & 36,000 & [745-6.87] & 36,000 & [738-5.63] & 36,000 \\
\hline 22 & TE 60 & 3 & [1569-26.25] & 36,000 & [1557-24.17] & 36,000 & [1586-23.25] & 36,000 \\
\hline 23 & & 4 & [1353-23.77] & 36,000 & {$[1345-23.51]$} & 36,000 & [1347-23.33] & 36,000 \\
\hline 24 & & 5 & [1258-21.25] & 36,000 & [1267-21.18] & 36,000 & [1283-22.25] & 36,000 \\
\hline 25 & TR 60 & 3 & 274 & 1233 & [274-5.61] & 36,000 & 274 & 45.26 \\
\hline 26 & & 4 & 207 & 2895 & 207 & 17,228 & 207 & 3685 \\
\hline 27 & & 5 & 189 & 567 & 189 & 4345 & 189 & 2508 \\
\hline 28 & TE 80 & 3 & & & & & [1935-30.72] & 36,000 \\
\hline 29 & & 4 & & & & & [1742-32.55] & 36,000 \\
\hline 30 & & 5 & & & & & [1526-25.85] & 36,000 \\
\hline
\end{tabular}

With respect to the solution times, direct comparison is not possible because the results in Dahl et al. [26] are obtained on a PC with a $2.4 \mathrm{GHz}$ Pentium IV processor and $768 \mathrm{MB}$ of RAM by using ILOG CPLEX 7.1. On the other hand, some inferences can be made from the results. The flow-based formulation cannot find optimal solutions for TE 40 instances within the running time (not specified exactly but stated to be more than one day). For the remaining instances, the solution times of HMST/SD2 are better on the average about 50 times implying that HMST/SD2 is likely to produce better solution times. The hop-indexed formulation can find optimal solutions for all instances except for two TE 60 instances (Pr. Id. 23 and 24). For the remaining problems, the hopindexed formulation is significantly superior implying that the hop-indexed formulation will produce better solution times. On the other hand, when the figures for TE 60 instances are considered, some striking observations are made. The results show that the LP relaxation bounds of the hop-indexed formulation are obtained in 2548, 23,402, and 73,300 s for Pr.Id. 22, 23, and 24, respectively, whereas (integer) feasible solutions for the same problems are obtained by HMST/SD2 just in seconds. Dahl et al. [26] state that, an upper cutoff value of 1354 has been generated after 7 days and no new upper cutoff value has been generated after 11 days of computation for Pr. Id. 23. However, for the same instance, upper cutoff values of 1370, 1355, and 1347 are obtained by HMST/SD2 at 2800, 4900, and 15,500 s, respectively. Further computational tests on TE 80 instances indicate similar results. Dahl et al. [26] report that the hop-indexed formulation can obtain LP relaxation bounds in 16,127 and 160,127 s for TE 80 instances with $p=3$ and 4 (Pr. Id. 28 and 29 in Table 2). The model is not run and hence no result is reported for $p=5$ (Pr. Id. 30). For the same problems, HMST/SD2 can obtain feasible solutions just in seconds. Moreoever, good upper cutoff values are obtained in a reasonable amount of time. For Pr. Id. 28 whose optimal objective function value is 1806 , the initial upper cutoff value of 3430 is improved to 1981 in 600 s. For Pr. Id. 29 whose known best upper cutoff value is 1631 , the initial value of 2344 is improved to 1742 at 30,000 s. Similarly, for Pr. Id. 30 whose known best upper cutoff value is 1500 , initial value of 1836 is improved to 1536 in $4500 \mathrm{~s}$. Note that these results are obtained by using the default settings of CPLEX and better results may be 
obtained by using different parameter settings that emphasize finding better upper cutoff values or improving the lower bounds [40].

The aforementioned results show that the LP bounds of HMST/ SD2 are significantly inferior to those of flow-based and hopindexed formulations. However, HMST/SD2 is attractive especially due to its compactness compared to the flow-based and hopindexed formulations as well as other models and ability to find good feasible solutions in a reasonable amount of time.

\section{Conclusion}

This paper develops new MTZ-based formulations for HMST. The first model HMST/SD is obtained by adapting ATSP model of Sherali and Driscoll [18] to formulate HMST and the other two models HMST/SD1 and HMST/SD2 are obtained by adding topology-enforcing and MTZ-related constraints offered by Akgün and Tansel [20] to the first model appropriately. Computational studies show that the HMST/SD does not dominate the MTZ-based model with the best LP bounds in the literature for all cases while HMST/SD1 and HMST/SD2 do on the average by $3.6 \%$ and $4.14 \%$, respectively. The new models do not produce solution times better than the best ones obtained by the MTZ-based models in the literature for the problems solved to optimality by all models. On the other hand, the results imply that HMST/SD2 is likely to produce better solution times for the harder, large-size instances. Comparison of HMST/SD2 to flow-based and hop-indexed formulations indicates that HMST/SD2 is inferior with respect to LP bounds. However, it is attractive due to its compactness and ability to find good feasible solutions in a short time within which even the LP relaxations of flow-based and hop-indexed formulations cannot be solved.

\section{Acknowledgements}

The author is grateful to two anonymous referees for providing constructive feedback that has helped improve in major ways the presentation of the material in the paper.

\section{References}

[1] Ahuja R, Magnanti T, Orlin J. Network flows: theory, algorithms and applications. Prentice-Hall; 1993.

[2] Woolston K, Albin S. The design of centralized networks with reliability and availability constraints. Computers and Operations Research 1988;15: 207-17.

[3] LeBlanc L, Chifflet J, Mahey P. Packet routing in telecommunication networks with path and flow restrictions. INFORMS Journal on Computing 1999;11: 188-97.

[4] Balakrishnan A, Altinkemer K. Using a hop-constrained model to generate alternative communication network design. ORSA Journal on Computing 1992;4:192-205.

[5] Gouveia L, Patricio P, Sousa A, Valadas R. MPLS over WDM network design with packet level QoS constraints based on ILP models. In: Proceedings of the INFOCOM 2003, 22nd annual joint conference of the IEEE computer and communications, IEEE Societies, vol. 1, April 2003. p. 576-86.

[6] Dahl G. The 2-hop spanning tree problem. Operations Research Letters 1998;23:21-6.

[7] Kruskal JB. On the shortest spanning subtree of a graph and the traveling salesman problem. Proceedings of the American Mathematics Society 1956;7(1):48-50.

[8] Prim R. Shortest connection networks and some generalizations. Bell System Technical Journal 1957;36:1389-401.

[9] Gouveia L. Using the Miller-Tucker-Zemlin constraints to formulate minimal spanning trees with hop constraints. Computers and Operations Research 1995;22:959-70.

[10] Manyem P, Stallmann M. Some approximation results in multicasting. Technical Report, North Carolina State University, 1996.

[11] Alfandari L, Paschos VTh. Approximating minimum spanning tree of depth 2. International Transactions in Operational Research 1999;6:607-22.
[12] Miller C, Tucker A, Zemlin R. Integer programming formulation of traveling salesman problems. Journal of Association for Computing Machinery 1960;7:326-9.

[13] Magnanti T, Wolsey L. Optimal trees. In: Ball MO, Magnanti TL, Monma CL, Nemhauser GL, editors. Network models, handbooks in operations research and management science, vol. 7. Amsterdam: North-Holland; 1995. p. 503-615.

[14] Dantzig G, Fulkerson D, Johnson S. Solution of a large scale traveling salesman problem. Operations Research 1954;2:393-410.

[15] Orman AJ, Williams HP. A survey of different integer programming formulations of the traveling salesman problem. Preprint Series \#OR101. Faculty of Mathematical Studies, University of Southampton, Southampton, England, 1999

[16] Desrochers M, Laporte G. Improvements and extensions to the MillerTucker-Zemlin subtour elimination constraints. Operations Research Letters 1991;10:27-36.

[17] Gouveia L, Pires JM. The asymmetric travelling salesman problem and a reformulation of the Miller-Tucker-Zemlin constraints. European Journal of Operational Research 1999;112:134-46.

[18] Sherali HD, Driscoll PJ. On tightening the relaxations of Miller-TuckerZemlin formulations for asymmetric traveling salesman problems. Operations Research 2002;50(4):656-69.

[19] Grötschel M, Padberg M. Polyhedral theory. In: Lawler EL, Lenstra JK, Rinnooy Kan A, Shymos DB, editors. The traveling salesman problem: a guided tour of combinatorial optimization. New York: John Wiley and Sons; 1985.

[20] Akgün İ, Tansel BÇ. New formulations via Miller-Tucker-Zemlin constraints for the hop-constrained minimum spanning tree problem, submitted for publication.

[21] Gouveia L. Multicommodity flow models for spanning trees with hop constraints. European Journal of Operational Research 1996;95:178-90.

[22] Gouveia L. Using variable redefinition for computing lower bounds for minimum spanning and Steiner trees with hop constraints. INFORMS Journal on Computing 1998;10:180-8.

[23] Gouveia L, Requejo C. A new lagrangian relaxation approach for the hopconstrained minimum spanning tree problem. European Journal of Operational Research 2001;132(3):539-52.

[24] Pirkul H, Soni S. New formulations and solution procedures for the hop constrained network design problem. European Journal of Operational Research 2003;148:126-40.

[25] Dahl G, Flatbert T, Foldnes N, Gouveia L. The jump formulation for the hopconstrained minimum spanning tree problem. Technical report, Centro de Investigaciao Operacional, Faculdade de Ciencias da Universidade de Lisboa, 2004.

[26] Dahl G, Gouveia L, Requeijo C. On formulations and methods for the hopconstrained minimum spanning tree problem. In: Resende MGC, Pardalos PM, Pardalos PM, editors. Handbooks of telecommunications. Springer; 2006. p. $493-515$.

[27] Kerivin H, Mahjoub AR. Design of survivable networks: a survey. Networks 2005;46(1):1-21.

[28] Gouveia L, Simonetti L, Uchoa E. Modeling hop-constrained and diameterconstrained minimum spanning tree problems as Steiner tree problems over layered graphs. Mathematical Programming 2010. URL < http://dx.doi.org/10. 1007/s10107-009-0297-2>.

[29] Hwang FK, Richards DS, Winter P. The steiner tree problems. Annals of discrete mathematics. North-Holland; 1992.

[30] Maculan N. The Steiner problem in graphs. Annals of Discrete Mathematics 1987;31:185-212.

[31] Gouveia L, Paiasa A, Sharmab D. Modeling and solving the rooted distanceconstrained minimum spanning tree problem. Computers and Operations Research 2008;35:600-13.

[32] Achuthan NR, Caccetta L, Caccetta P, Geelen JF. Algorithms for the minimum weight spanning tree with bounded diameter problem. In: Phua $\mathrm{PKH}$ Wang CM, Yeong WY, Leong TY, Loh HT, Tan KC, Chou FS, editors. Optimization techniques and applications, vol. 1. World Scientific Publishing; 1992. p. 297-304.

[33] Gouveia L, Magnanti TL. Network flow models for designing diameterconstrained minimum-spanning and Steiner trees. Networks 2003;41(3): 159-73.

[34] Gruber M, Raidl GR. A new 0-1 ILP approach for the bounded diameter minimum spanning tree problem. In: Proceedings of the 2nd international network optimization conference, Lisbon, 2005. p. 178-85.

[35] Santos AC, Lucena A, Ribeiro CC. Solving diameter constrained minimum spanning tree problems in dense graphs. In: Experimental and efficient algorithms, vol. 3059/2004 of Lecture notes in computer science. Berlin: Springer; 2004. p. 458-67.

[36] Lawler E, Lenstra J, Kan A, Shmoys D. The traveling salesman problem. New York: John Wiley and Sons; 1992.

[37] Padberg M, Sung T. An analytical comparison of different formulations of the traveling salesman problem. Mathematical Programming 1992;52:315-57.

[38] Nemhauser GL, Wolsey LA. Integer and combinatorial optimization. New York: John Wiley \& Sons; 1999.

[39] Akgün İ, Tansel BÇ. Min-degree constrained minimum spanning tree problem: new formulation via Miller-Tucker-Zemlin constraints. Computers and Operations Research 2010;37(1):72-82.

[40] ILOG/CPLEX 9.0, Reference manual, 1997. 